nuclear layer oedema and thinning of the ganglion cell layer during follow-up (Fig. 2). At presentation magnetic resonance imaging (MRI) showed subtle white matter changes in the occipital lobes.

Fludarabine-related visual loss is well documented and our patients had several classic features. Diagnosis must be made based on history, as initial examination and OCT are normal. Fludarabine must be stopped when toxicity is suspected and investigated with ERG and brain MRI. This report is the first to include OCT. Serial OCTs during follow-up show the evolution of various retinal changes (see above), most of which have previously been shown via histopathology in case reports. There is currently no good quality evidence to guide treatment.

\section{Compliance with ethical standards}

Conflict of interest The authors declare that they have no conflict of interest.
Publisher's note: Springer Nature remains neutral with regard to jurisdictional claims in published maps and institutional affiliations.

\section{References}

1. Warren RP, Jr, Berman E. Phase I and II study of fludarabine phosphate in leukemia: therapeutic efficacy with delayed central nervous system toxicity. J Clin Oncol. 1986;4:74-9.

2. Sorensen, et al. Treatment of refractory chronic lymphocytic leukemia with fludarabine phosphate via the Group C Protocol Mechanism of the National Cancer Institute: five-year follow-up report. J Clin Oncol. 1997;15:458-65.

3. Bishop, et al. Rapid vision loss associated with fludarabine administration. Retina. 2010;30:1272-7.

4. Ding, et al. Ocular toxicity of fludarabine: a purine analog. Expert Rev Ophthalmol. 2008;3:97-109.

5. Lee, et al. Clinical and Imaging Features of Fludarabine Neurotoxicity. J Neuroophthalmol. 2010;30:37-41.

\title{
Needles as a source of silicone oil during intravitreal injection
}

\author{
Gustavo Barreto Melo $\mathbb{1}^{1,2} \cdot$ Geoffrey Guy Emerson ${ }^{3} \cdot$ Acácio Alves Souza Lima Filho ${ }^{2} \cdot$ Shoko Ota ${ }^{4}$ Maurício Maia ${ }^{2}$
}

Received: 20 December 2018 / Accepted: 21 December 2018 / Published online: 13 February 2019

(c) The Royal College of Ophthalmologists 2019

\section{Introduction}

The presence of silicone oil (SO) droplets in the vitreous, even though frequently considered harmless, has been reported in $0.03-1.7 \%$ of eyes receiving intravitreal injections [1].

The source of these droplets is supposedly the syringes, since they are coated with $\mathrm{SO}$ in order to facilitate the

Gustavo Barreto Melo

gustavobmelo@yahoo.com.br

Hospital de Olhos de Sergipe, Aracaju, SE, Brazil

Federal University of São Paulo, São Paulo, SP, Brazil

Retina Center of Minnesota, Minneapolis, MN, USA

4 Chemical Analysis Laboratory, Center for Chemistry and Manufactured Goods, Institute for Technological Research, São Paulo, Brazil



Fig. 1 BD PrecisionGlide needles. a 30-gauge needle; b 26-gauge needle 

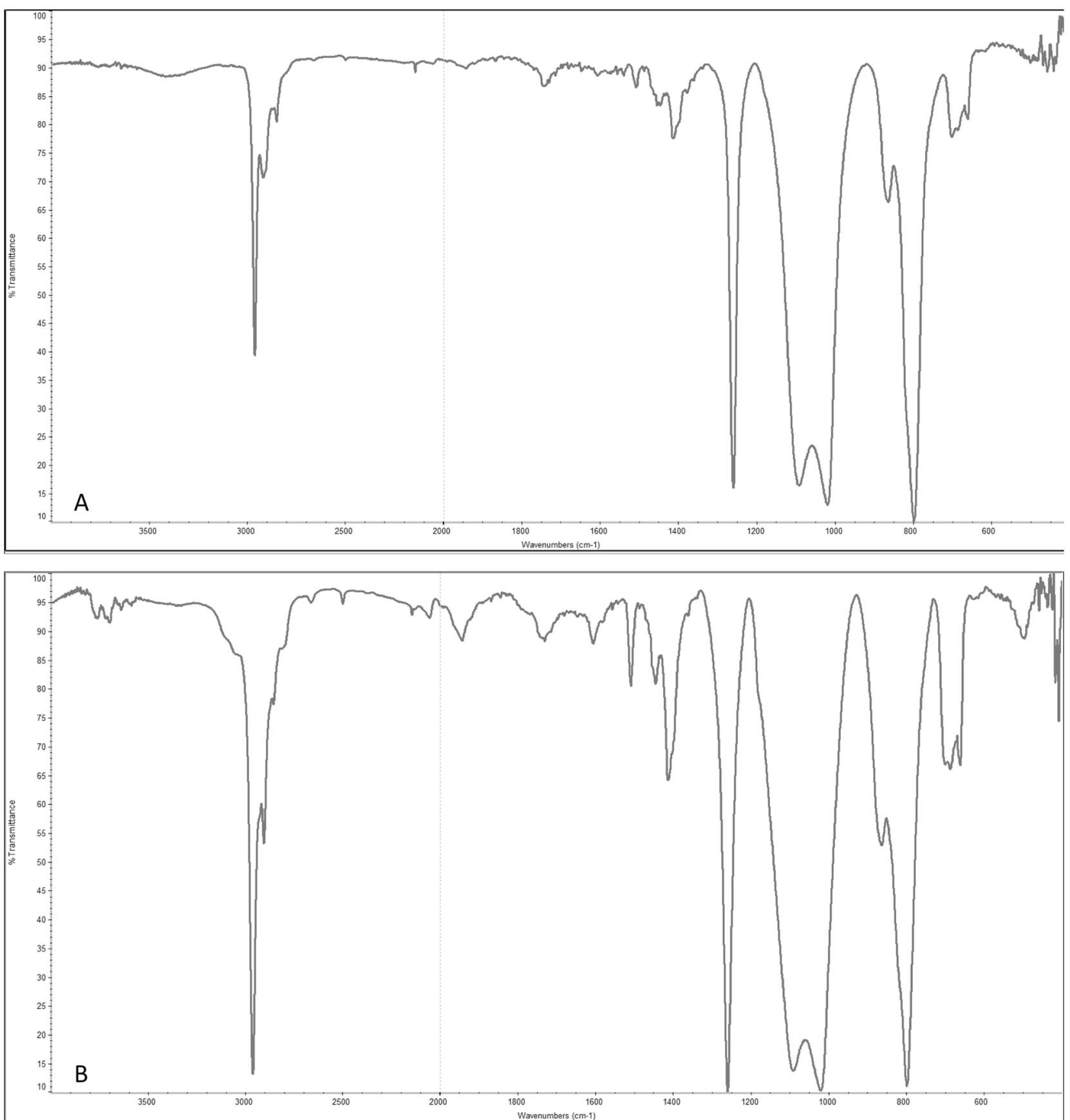

Fig. 2 Fourier-transform infrared spectroscopy showing characteristic bands corresponding to polysiloxane, i.e., silicone oil, in both BD PrecisionGlide needles. a 30-gauge needle; b 26-gauge needle

gliding of the plunger [2]. However, it is uncertain the role the needles might have. Therefore, this study assessed whether two models of needles contained SO.

\section{Materials and methods}

One hundred samples each of two models of needles (Fig. 1) from the same manufacturer were studied: BD PrecisionGlide 26-gauge (Becton-Dickinson, Curitiba, Brazil, lot \# 8234979, ref \# 300110) and 30-gauge needle (lot \# 8165866, ref \# 990193). The metallic part of the needle was removed from the plastic hub. Groups of 50 samples were immersed in $50 \mathrm{~mL}$ of chloroform (99.8\%, Anidrol, São Paulo, Brazil) for $30 \mathrm{~min}$ under ultrasound, $40 \mathrm{kHz}$ (USC-4800, Unique, São Paulo, Brazil). Then the chloroform was evaporated in a water bath (TE-056, Tecnal, São Paulo, Brazil) followed by an oven (Q314M212, Quimis, São Paulo, Brazil) at $105^{\circ} \mathrm{C}$. After cooling, 
the mass of the extract was verified by an analytical scale (AB204-S, Mettler Toledo, Switzerland). The dry extract was assessed by Fourier-transform infrared spectroscopy (Nicolet iS10, Thermo Electron Scientific Instruments, Madison, WI), with band ranging from $4000 \mathrm{~cm}^{-1}$ to $400 \mathrm{~cm}^{-1}$, resolution of $4 \mathrm{~cm}^{-1}$, and 32 scans.

\section{Results}

Analysis of the material indicated the presence of polysiloxane (i.e., SO) in both needles (Fig. 2). The following characteristic bands were found: $2960 \mathrm{~cm}^{-1}$, stretching vibrations of $\mathrm{CH}_{3}$ in $\mathrm{Si}-\mathrm{CH}_{3} ; 1260 \mathrm{~cm}^{-1}$, bending vibrations of $\mathrm{CH}_{3}$ in $\mathrm{Si}-\mathrm{CH}_{3} ; 1090 \mathrm{~cm}^{-1}$ and $1020 \mathrm{~cm}^{-1}$, asymmetric stretching vibrations of $\mathrm{Si}-\mathrm{O}-\mathrm{Si} ; 800 \mathrm{~cm}^{-1}$, stretching vibrations of $\mathrm{Si}-\mathrm{C}$ in $\mathrm{Si}_{-}-\mathrm{CH}_{3}$ [3].

Both 30- and 26-gauge needles contained on average 1 and $1.5 \mathrm{mg}$, respectively, of polysiloxane for every 50 samples, yielding an estimate of 20 and $30 \mu \mathrm{g}$, respectively, per unit.

\section{Discussion}

SO is a lubricant commonly used in medical catheters, syringes, and needles to aid comfort during insertion and control during the delivery of medication. The main concern about $\mathrm{SO}$ in the eye is that it may cause symptomatic floaters. A report from the American Society of Retina Specialists showed that $5.2 \%$ of US retina specialists have performed vitrectomy to remove SO droplets after intravitreal injection of bevacizumab [4]. This is undesirable due to the inherent risks of invasive procedures.

Our suspicion for the presence of SO in needles follows from a separate study where we identified rare SO droplets in fluid ejected from a silicone-free syringe via a BD PrecisionGlide needle (unpublished data). The current analysis confirms that $\mathrm{SO}$ is present in two needle models; in other words, the use of silicone-free syringes does not completely eliminate the risk of SO droplets in the vitreous so long as the needle contains SO.

In conclusion, ophthalmologists should be aware that needles tend to be coated with SO and there is a potential risk of displacing some droplets into the eye.

Funding EyePharma (São Paulo, Brazil) supported this study.

Author contributions All authors conceived and/or designed the work that led to the submission, acquired data, and/or played an important role in interpreting the results, drafted or revised the manuscript, approved the final version, and agreed to be accountable for all aspects of the work.

\section{Compliance with ethical standards}

Conflict of interest The authors declare that they have no conflict of interest.

Publisher's note: Springer Nature remains neutral with regard to jurisdictional claims in published maps and institutional affiliations.

\section{References}

1. Khurana RN, Chang LK, Porco TC. Incidence of presumed silicone oil droplets in the vitreous cavity after intravitreal bevacizumab injection with insulin syringes. JAMA Ophthalmol. 2017;135:800-3.

2. Funke S, Matilainen J, Nalenz H, Bechtold-Peters K, Mahler HC, Friess W. Analysis of thin baked-on silicone layers by FTIR and 3D-laser scanning microscopy. Eur J Pharm Biopharm. 2015;96:304-13. https://doi.org/10.1016/j.ejpb.2015.08.009

3. Chamerski K, Lesniak M, Sitarz M, Stopa M, Filipecki J. An investigation of the effect of silicone oil on polymer intraocular lenses by means of PALS, FT-IR and Raman spectroscopies. Spectrochim Acta A Mol Biomol Spectrosc. 2016;167:96-100. https://doi.org/10.1016/j.saa.2016.05.036

4. Stone TW, editor. ASRS 2018 preferences and trends membership survey. Chicago, IL: American Society of Retina Specialists; 2018. https://www.asrs.org/content/documents/_2018-pat-survey-resultsfor-website.pdf. Accessed 29 Aug 2018. 